\title{
Structure-Properties Relationship in Polymers with Condensed Aromatic Rings. I. Structure-Thermal Properties of New Polyketanils
}

\author{
Danuta SEcK \\ Institute of Polymer Chemistry, Polish Academy of Sciences, \\ P.O. Box 49, 41-800 Zabrze, Poland.
}

(Received January 22, 1980)

\begin{abstract}
A series of new polyketanils, containing naphthyl rings in side chains having the structure

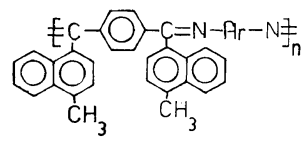

was obtained and a relationship between the structure of the diamine used and the thermal stability of the resultant polymer was found to exist. The influence of the conditions of synthesis on the yield and properties of the new polyketanils was also determined.

KEY WORDS Polyketanil / Aromatic Diketone / Thermal Stability /

Polycondensation /
\end{abstract}

Investigations on the relationship between the thermal stability of polymers and their chemical structures have shown that one of the factors determining their thermal stability, apart from the energy of interatomic bonds, is the resonance stabilization of the polymer chain.

The above relationship was confirmed in series of thermostable polymers containing conjugated aromatic rings, such as polyparaphenylenes, polybenzimidazoles, and others.

It is to be expected that the $\mathrm{C}=\mathrm{N}$ azomethine bonds incorporated in the polymer chain along with aromatic rings should also form a conjugated system which determines the thermal stability of polymers. Aromatic azomethine polymers can be obtained in the reaction of aromatic dicarbonyl compounds with aromatic diamines, or from monomers containing carbonyl and amine groups in the molecule.

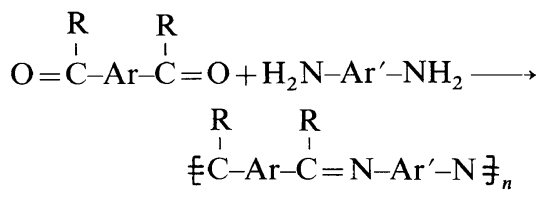<smiles>[R][C][14N][14CH2][14CH2][14C]([R])=O</smiles>

When using a dialdehyde with a carbonyl group as a monomer in the reaction, (i.e., when $\mathrm{R}=\mathrm{H}$ ), Schiff polybases are obtained. These Schiff polybases have been described by many authors as polymers possessing very high thermal stability. ${ }^{1-8}$ Only a few works have been published on the synthesis of polymers involving the use of a diketone as a dicarbonyl compound..$^{9-13}$ Up until now, only $p$ diacetylbenzene and $p$-dibenzoylbenzene or $m$ dibenzoylbenzene have been used as diketones yielding polyketanils in the reaction with diamines. Their thermal stability depends on the structure of the diketone as well as on that of the diamine employed. Thus it seems of interest to undertake an investigation on the use of new aromatic diketones in synthesizing polyketanils. The well-known influence of side groups in the chain on the thermal stability and on the solubility of polymers prompted us to use new aromatic diketones having naphthyl structures to synthesize new polyketanils.

The structures for the new diketones are presented in Figure 1. 


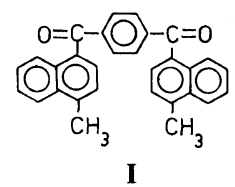

p-Bis(4-methyl-1-naphthoyl)benzene

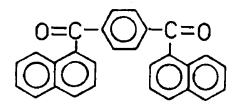

II

$p$-Bis(1-naphthoyl)benzene

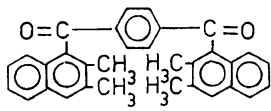

III

p-Bis(2,3-dimethyl-1-naphthoyl)benzene

Figure 1. The structure of the diketones.

This paper reports some results related to the dependence of the diamine structure and the conditions of synthesis of the polyketanils from diketone I on the thermal stability of polymers.

\section{EXPERIMENTAL}

\section{Aromatic Diketones}

The new aromatic diketones were obtained by reacting of terephthaloyl chloride with naphthalene or a methyl derivative of naphthalene in the presence of aluminum chloride. In the case of using naphthalene and 2,3-dimethylnaphthalene, the reaction was carried out in a solution of dichloromethane, while in the case of 1-methylnaphthalene, the hydrocarbon served also as a solvent.

\section{p-Bis(4-methyl-1-naphthoyl)benzene (I)}

To $50 \mathrm{ml}$ of 1 -methynaphthalene, containing $20.4 \mathrm{~g}(0.1 \mathrm{~mol})$ of terephthaloyl chloride there was added slowly with vigorous stirring $26.6 \mathrm{~g}(0.2 \mathrm{~mol})$ of anhydrous $\mathrm{AlCl}_{3}$ for $45 \mathrm{~min}$.

The reaction was carried out at $20^{\circ} \mathrm{C}$ over a period of $10 \mathrm{~h}$ and then for $10 \mathrm{~h}$ at $80^{\circ} \mathrm{C}$. To the reaction mixture was added $50 \mathrm{ml}$ of acetone and this whole mixture was poured into $100 \mathrm{ml}$ of $10 \%$ $\mathrm{HCl}$ containing crushed ice. $100 \mathrm{ml}$ of acetone were added to the crude product after being washed with water.
The precipitate formed was filtered off and then crystallized from xylene. The product yield was $55 \%$.

p-Bis (1-naphthoyl)benzene (II)

To a solution of $5.12 \mathrm{~g}(0.04 \mathrm{~mol})$ of naphthalene and $4.08 \mathrm{~g}(0.02 \mathrm{~mol})$ of terephthaloyl chloride in $50 \mathrm{ml}$ of dichloromethane there was slowly added in portions, with vigorous stirring, $5.32 \mathrm{~g}(0.04 \mathrm{~mol})$ of anhydrous $\mathrm{AlCl}_{3}$ over a period of $30 \mathrm{~min}$. The reaction was continued at $20^{\circ} \mathrm{C}$ for $20 \mathrm{~h}$.

Following completion of the synthesis, the reaction solution was poured into $100 \mathrm{ml}$ of $10 \% \mathrm{HCl}$ containing crushed ice, and then washed with water. The solvent was evaporated and the crude product was crystallized from xylene.

The yield of the reaction was $15 \%$.

\section{p-Bis(2,3-dimethyl-1-naphthoyl)benzene (III)}

The synthesis of this compound was carried out in the same way as II, using 2,3-dimethylnaphthalene instead of naphthalene. The product yield was $50 \%$.

\section{The Model Compound}

In order to examine whether or not the diketone I could react with aromatic amines, a model compound was synthesized by condensing the respective diketone with aniline.

\section{The Model Compound from the Diketone I}

$2.07 \mathrm{~g}$ of diketone I were heated along with $20 \mathrm{ml}$ of aniline in the presence of $0.1 \mathrm{~g}$ of $p$ toluenesulphonic acid at $184^{\circ} \mathrm{C}$ over a period of $20 \mathrm{~h}$. The reaction solution was then poured into $50 \mathrm{ml}$ of ethanol, the precipitate filtered off and the crude product was crystallized from xylene.

The yield of the reaction was $75 \%$.

\section{Polymers}

The polycondensation of diketone I with different

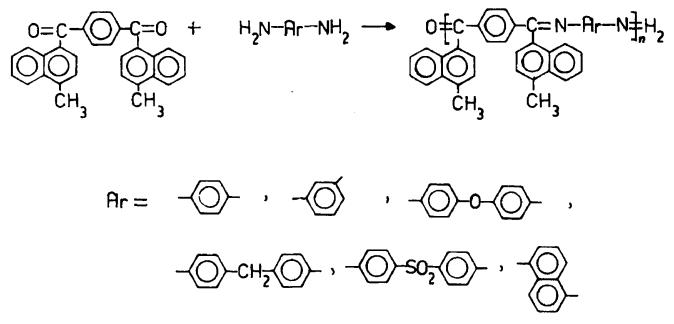

Figure 2. Scheme of the reaction. 
amines, proceeding according to the scheme presented in Figure 2, was carried out in various solvents and at different temperatures in order to study the influence of varying reaction conditions on the thermal stability of the polyketanils obtained.

\section{Polycondensation in Decalin}

$0.01 \mathrm{~mol}$ of diketone $\mathbf{I}$ and $0.01 \mathrm{~mol}$ of a diamine were heated in $100 \mathrm{ml}$ of decalin at the boiling point in the presence of $0.2 \mathrm{~g}$ of $p$-toluenesulphonic acid under a blanket of argon. After $48 \mathrm{~h}$ of heating, the polymer was filtered off and then extracted with ethanol in the Soxhlet apparatus over a period of $12 \mathrm{~h}$.

\section{Polycondensation in a m-Cresol- $\mathrm{P}_{2} \mathrm{O}_{5}$ Mixture}

To $30 \mathrm{~g}$ of $\mathrm{m}$-cresol were added, in portions, $8.5 \mathrm{~g}$ of $\mathrm{P}_{2} \mathrm{O}_{5}$ and the resulting slurry was heated at $140^{\circ} \mathrm{C}$ for $2 \mathrm{~h}$. After cooling, $0.005 \mathrm{~mol}$ of diketone $\mathbf{I}$ and $0.005 \mathrm{~mol}$ of diamine were added to the mixture which was then heated at $130^{\circ} \mathrm{C}$ or $160^{\circ} \mathrm{C}$ for a period of about $48 \mathrm{~h}$. The reaction mixture was then poured into $700 \mathrm{ml}$ of ethanol containing $25 \mathrm{ml}$ of triethylamine.

The precipitated polymer was filtered off and then extracted with ethanol in the Soxhlet apparatus for $12 \mathrm{~h}$.

\section{Polycondensation in Poly (phosphoric Acid)}

Poly(phosphoric acid) (PPA) was freshly prepared by the addition of $4.4 \mathrm{~g}$ of $\mathrm{P}_{2} \mathrm{O}_{5}$ to $10.0 \mathrm{~g}$ of $85 \%$ phosphoric acid.

This slurry was stirred under an argon atmosphere and heated at $160^{\circ} \mathrm{C}$ for $6 \mathrm{~h}$. The clear homogeneous PPA was cooled to room temperature and $0.005 \mathrm{~mol}$ of diketone $\mathbf{I}$ and $0.005 \mathrm{~mol}$ of diamine were added to it. The mixture was heated at $160^{\circ} \mathrm{C}$ for $20 \mathrm{~h}$ and then at $180^{\circ} \mathrm{C}$ for $20 \mathrm{~h}$. The reaction mixture after cooling to room temperature was poured into $500 \mathrm{ml}$ of ethanol containing $100 \mathrm{ml}$ of water. The resulting polymer was filtered, washed with water, and then extracted with ethanol in the Soxhlet apparatus for $12 \mathrm{~h}$.

\section{Polycondensation in Concentrated $\mathrm{H}_{2} \mathrm{SO}_{4}$}

A mixture of $0.005 \mathrm{~mol}$ of diketone $\mathbf{I}$ and 0.005 mol of a diamine in $15 \mathrm{ml}$ of concd $\mathrm{H}_{2} \mathrm{SO}_{4}$ was left at $20^{\circ} \mathrm{C}$ for a period of 8 days and then maintained at a temperature of $60^{\circ} \mathrm{C}$ for 8 subsequent days. The reaction solution was then poured into $150 \mathrm{ml}$ of water containing crushed ice, and the resulting precipitate was washed with water, and extracted with ethanol in the Soxhlet apparatus for $12 \mathrm{~h}$.

\section{Measurements}

The infrared spectra were recorded in nujol on the UR-20 (Zeiss) apparatus.

The NMR spectra were recorded on a XL-100 Varian spectrometer for samples dissolved in deuterated chloroform, using HMDSO as the internal standard.

A mass analysis was made on a LKB 9000 spectometer, whose electron-beam energy was equal to $70 \mathrm{eV}$ at the source temperature of $290^{\circ} \mathrm{C}$.

Thermogravimetric measurements were performed in air at $1 \mathrm{~atm}$ on a derivatograph (F. Paulik, J. Paulik, and L, Erdey), at a heating rate of $10^{\circ} \mathrm{C}$ $\min ^{-1}$ using the Pt-Pt-Rh thermocouple.

\section{DISCUSSION}

The structure of new diketones (presented in Table I) was confirmed by mass, infrared (IR), and nuclear magnetic resonance (NMR) spectroscopy methods. The infrared spectra showed the presence of a strong band of stretching vibrations $\mathrm{C}=\mathrm{O}$ at $1650 \mathrm{~cm}^{-1}$, bending vibrations $\mathrm{C}-\mathrm{C}=\mathrm{O}$ at 1250 $\mathrm{cm}^{-1}$, and absorption bands characteristic of the phenyl and naphthyl structures. In the NMR spectra, a singlet characteristic could be observed for the four hydrogen atoms in the phenyl ring, and a multiplet derived from the hydrogen atoms in the naphthyl rings. Moreover, in the case of the diketone $\mathbf{I}$, a singlet derived from the methyl group was observed, and for the diketone III, a doublet due to the presence of two methyl groups in different positions in the naphthyl ring, could be observed.

It can be seen the large differences in yields of the diketones I and III in comparison with the yield of the diketone II. It is probably due to the inductive effect of the methyl groups present in the 1methylnaphthalene and in the 2,3-dimethylnaphthalene on the reactivities of these hydrocarbons toward the terephthaloyl chloride.

The presence of two methyl groups in the diketone III was also found to have an influence on the fragmentation of its molecule subjected to the action of an electron beam.

In the mass spectrum of the diketone I, the peak 
D. SẸK

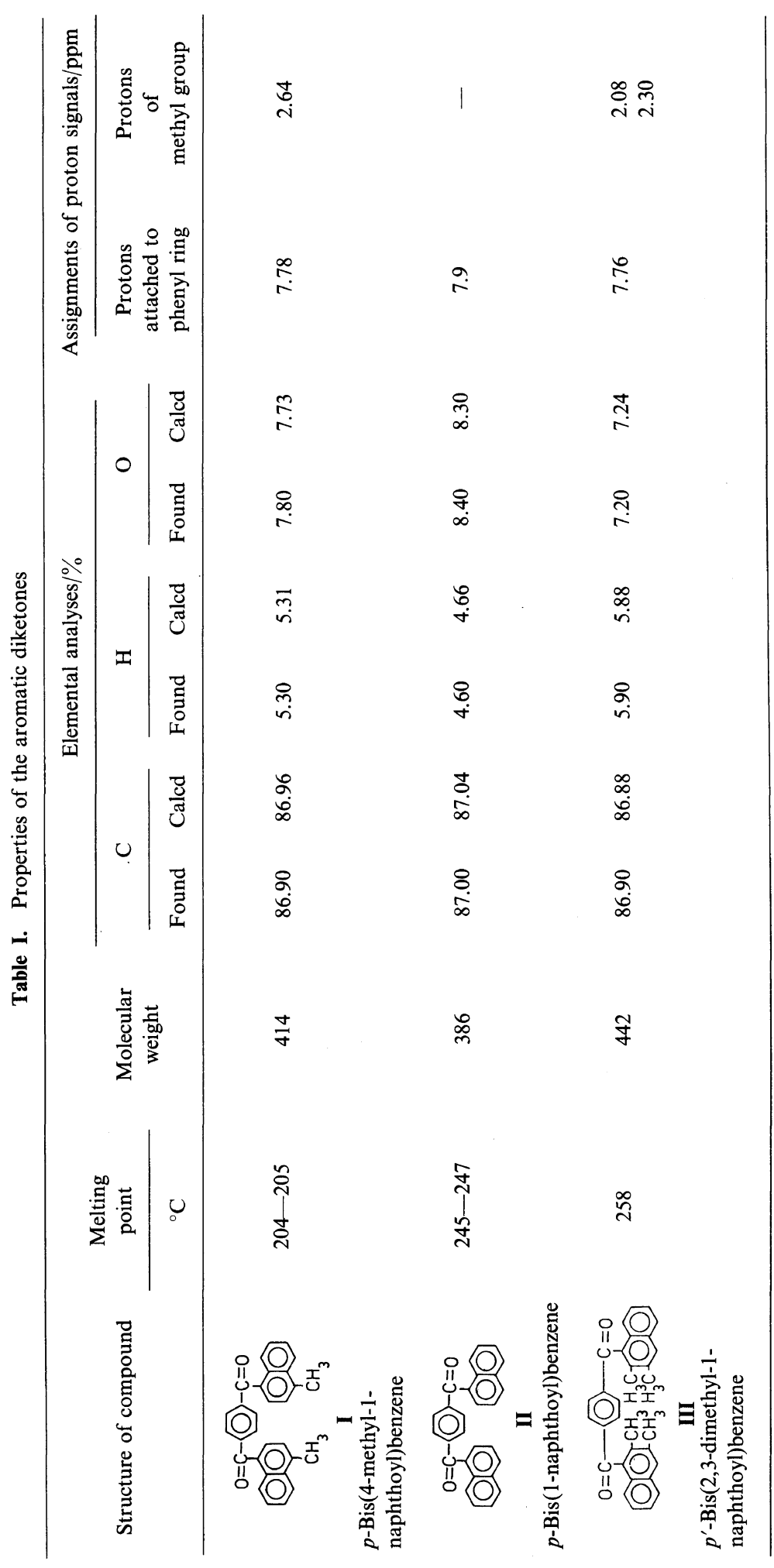


Structure and Thermal Properties of New Polyketanils

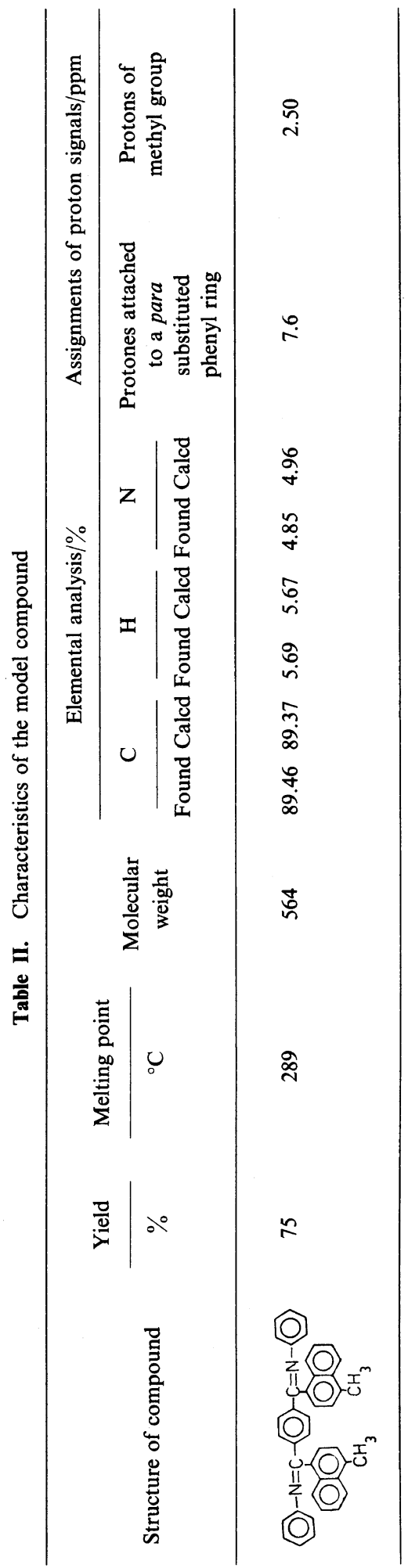

Polymer J., Vol. 13, No. 1, 1981 
D. SEK

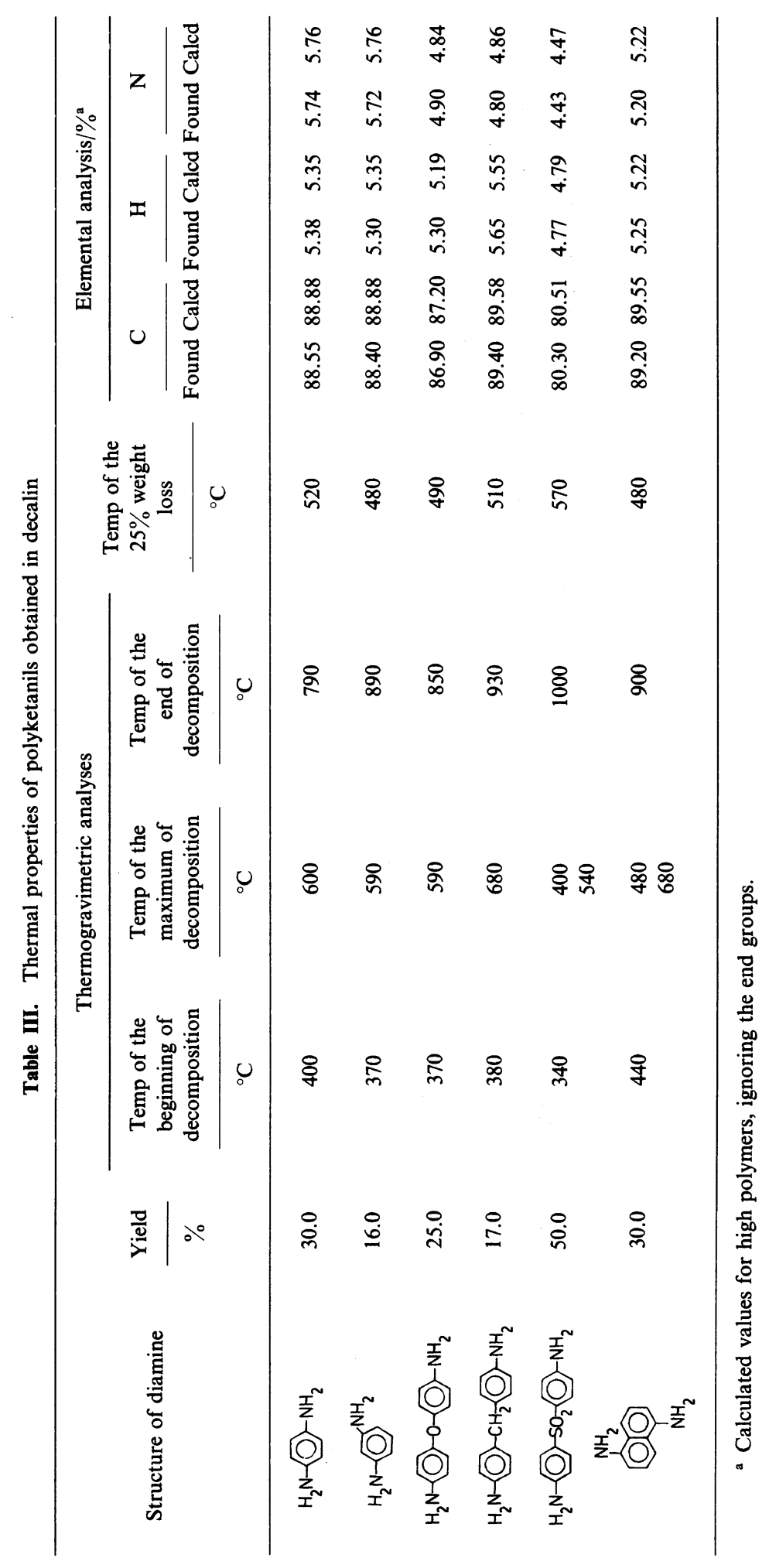


Structure and Thermal Properties of New Polyketanils

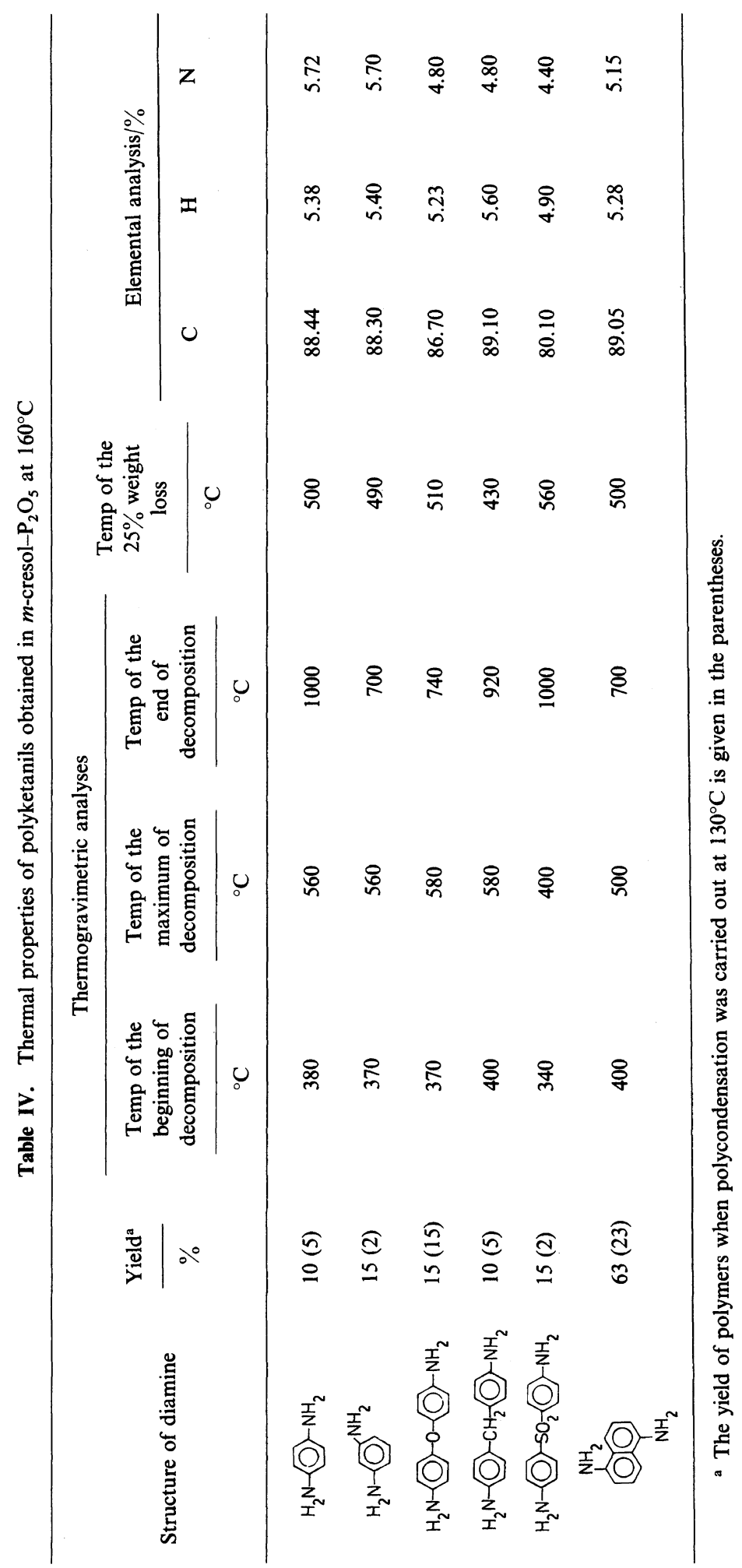

Polymer J., Vol. 13, No. 1, 1981 
D. SEK

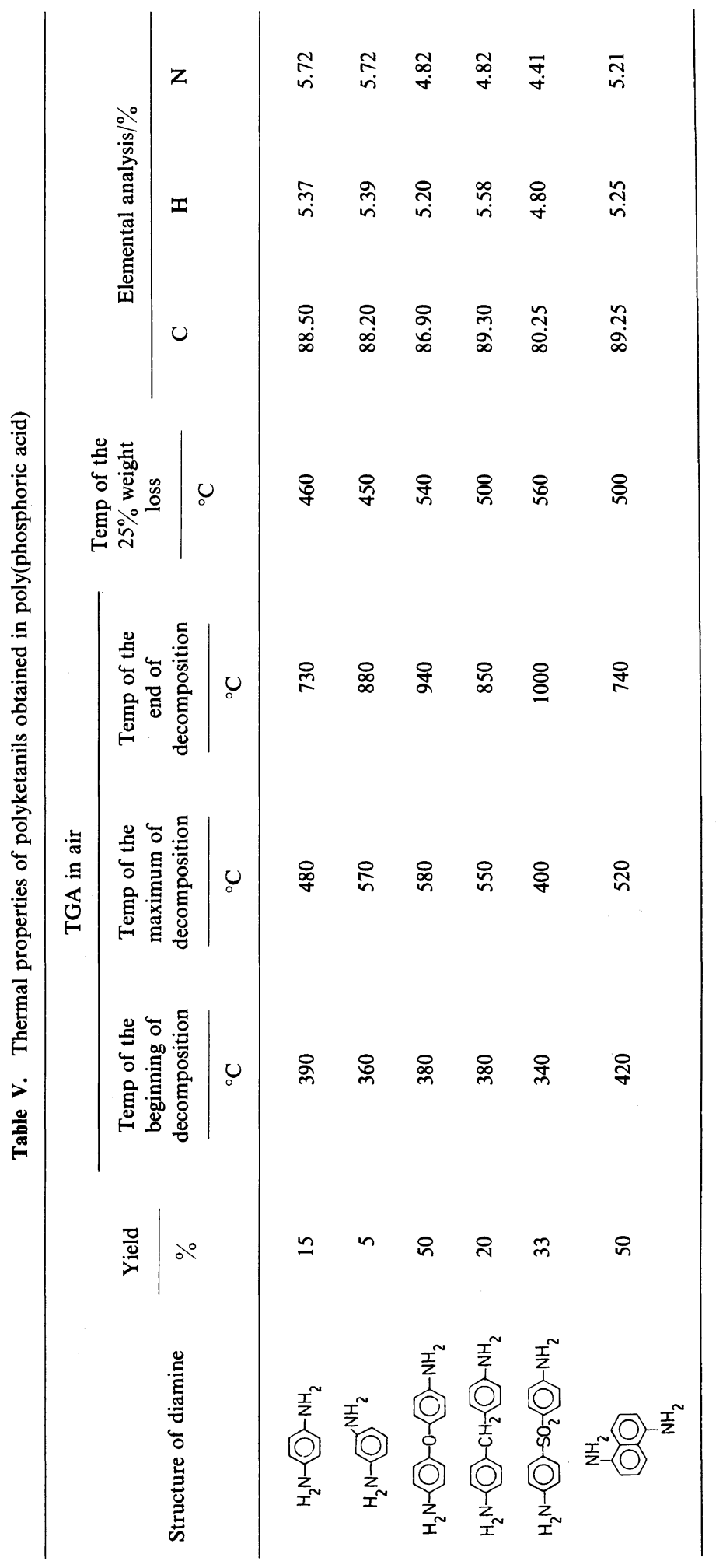


intensity at $m / e=169$ due to the fragment $\mathrm{C}_{11} \mathrm{H}_{9} \mathrm{CO}^{+}$was higher than the peak intensity at $m / e=141$ due to the fragment $\mathrm{C}_{11} \mathrm{H}_{9}{ }^{+}$.

The same dependence was observed in the mass spectrum of the diketone II, where the peak intensity at $m / e=155$ due to the fragment $\mathrm{C}_{10} \mathrm{H}_{7} \mathrm{CO}^{+}$was higher than the peak intensity at $m / e=127$ due to the fragment $\mathrm{C}_{10} \mathrm{H}_{7}{ }^{+}$.

On the other hand, in the mass spectrum of the diketone III the peak at $m / e=155$ due to the fragment $\mathrm{C}_{10} \mathrm{H}_{11}{ }^{+}$had a higher intensity than the peak at $m / e=183$ due to the fragment $\mathrm{C}_{12} \mathrm{H}_{11} \mathrm{CO}^{+}$.

The results of mass spectrometric analysis and those of IR and NMR analyses confirmed the structure of the model compound obtained (structure is shown in Table II).

A strong absorption band at $1610 \mathrm{~cm}^{-1}$, which is characteristic of the $-\mathrm{C}=\mathrm{N}$ - bond, was observed in the infrared spectrum of the model compound in addition to the absorption bands characteristic of the phenyl and naphthyl structures.

The NMR spectrum consisted of a peak due to the four hydrogen atoms in the phenyl-ring substituted in the para position, a multiplet in the absorption range characteristic of the remaining aromatic protons, and a methyl group peak.

The condensation of the diketone I with different aromatic amines was carried out in various solvents, (decalin, $m$-cresol, PPA, and concd $\mathrm{H}_{2} \mathrm{SO}_{4}$ ), at different temperatures as to determine the effect of these factors on the yield and thermal stability of the polymers obtained.

Analysis of the infrared spectra of the polyketanils obtained showed the presence of an absorption band at $1610 \mathrm{~cm}^{-1}$, characteristic of the $-\mathrm{C}=\mathrm{N}$ - arrangement, and a weak absorption band at $1650 \mathrm{~cm}^{-1}$ derived probably from carbonyl groups situated at chain ends.

There could also be observed a weak absorption in the range $3200-3500 \mathrm{~cm}^{-1}$, and this absorption is known to be characteristic of amino groups.

The results of the elemental analysis of the polyketanils show that the amount of carbon, hydrogen, and nitrogen are rather close to the calculated values for a high polymer whose number of end groups is considered to be negligible. An exact determination of the molecular weight was not possible because of the poor solubility of the polyketanils.

D'Alelio and coworkers ${ }^{6}$ used the results of the elemental analysis for calculating the approximate degree of polycondensation but this method seems to be more applicable to low-molecular-weight oligomers.

The polyketanils obtained in decalin did not melt until the decomposition temperature had been reached (Table III).

The thermogravimetric analysis performed in air indicated that the polyketanils produced in decalin exhibit a very high thermal stability, and that temperatures marking the beginning of decomposition depend on the structure of the diamine used.

The polyketanil from 1,5-naphthylenediamine had the highest thermal stability the temperature at which it started decomposing being equal to $440^{\circ} \mathrm{C}$. Also, the polyketanil from 1,4-phenylenediamine had a high thermal stability (decomposition temp. $400^{\circ} \mathrm{C}$ ).

Disturbance of the symmetry of the polymer chain due to the 1,3-phenylenediamine causes the temperature of which decomposition starts to decrease to $310^{\circ} \mathrm{C}$. The introduction of bridges $(-\mathrm{O}-$, $\left.-\mathrm{CH}_{2}-,-\mathrm{SO}_{2}-\right)$ between the phenyl rings in the diamines was found to result in a decrease in thermal stability. Although free pairs of electrons of the oxygen atom of the diaminodiphenyl ether incorporated into the polymer chain might possibly participate in conjugation with $\pi$ electrons from rings in polymer chain to give pseudo conjugated structures (as in the case of a $-\mathrm{CH}_{2}$ - group positioned between rings), ${ }^{5}$ the above-mentioned polymers undergo degradation at temperatures lower than those at which the polyketanils derived from symmetrical diamines without bridges start decomposition. Polymers containing the naphthyl structure were found to exhibit a thermal stability higher than those with the phenyl-ring system in the main chain. Among the polyketanils obtained in decalin, the polymer from 4,4'-diaminodiphenyl sulphone had the lowest temperature for the start of decomposition. Irrespective of the structure of the diamine used, the polyketanils obtained in decalin were only partially soluble in concd $\mathrm{H}_{2} \mathrm{SO}_{4}$ and dimethyl sulfoxide (DMSO).

An increase in the yield of the condensation reaction was found to take place with an increase in temperature in the case of polyketanils obtained in a $m$-cresol- $\mathrm{P}_{2} \mathrm{O}_{5}$ mixture (Table IV), the conversion at $130^{\circ} \mathrm{C}$ being very low. Yet in spite of the reaction 
temperature, the obtained polyketanils did not melt before reaching the temperature at which decomposition started.

The only exception was the polyketanil from $4,4^{\prime}$ diaminodiphenyl ether obtained at $130^{\circ} \mathrm{C}$ in a mixture of $m$-cresol and $\mathrm{P}_{2} \mathrm{O}_{5}$, which had a melting point of $195-210^{\circ} \mathrm{C}$.

The thermogravimetric analysis performed for polymers obtained at $160^{\circ} \mathrm{C}$ showed that temperatures marking the start of decomposition differed but slightly from those found for polyketanils obtained in decalin (Table III).

Polyketanils obtained in poly(phosphoric acid) (Table V) were also infusible and the temperatures for the start of decomposition were close to those found for polymers obtained in decalin and in $\mathrm{m}$ cresol.

The solubility of the new polymers depended on the reaction conditions. The polyketanils obtained in $m$-cresol were soluble in DMSO and partially soluble in tetrachloroethane, while the polyketanils obtained in decalin and in poly(phosphoric acid) were only partially soluble in DMSO and concd $\mathrm{H}_{2} \mathrm{SO}_{4}$.

When the reaction was conducted in concd $\mathrm{H}_{2} \mathrm{SO}_{4}$, a precipitate was formed in the reaction mixture after 8 days of thermostating at $60^{\circ} \mathrm{C}$. The polymers were obtained in yields of about $5 \%$, were insoluble and did not melt until the start of the decomposition temperature had been reached. A relatively strong IR absorption band was found to be present at $1650 \mathrm{~cm}^{-1}$, thus confirming our assumption that low-molecular-weight oligomers were obtained in this case.

\section{CONCLUSIONS}

The thermal stability of the new polyketanils containing naphthyl rings in side chains was found to depend on the structure of the diamine used. The symmetrical and possibly the most remote positioning of the functional groups in the diamine $(1,4-$ phenylenediamine, 1,5-naphthylenediamine) was observed to result in an increase of the thermal stability of the polyketanils, whose thermal stability was higher than that of polymers obtained from diamines having an asymmetrical arrangement of the functional groups, (1,3-phenylenediamine) or having bridges between the rings.

It was observed that the thermal stability of the polyketanils investigated depended but slightly on the reaction conditions applied.

The condensation reaction yield was found to depend on the reaction temperature employed.

\section{REFERENCES}

1. A. D. Delman, A. A. Stein, and B. B. Simms, $J$. Macromol. Sci., Chem., A1, 147 (1967).

2. E. Dyer and C. C. Anderson, J. Polym. Sci., A-1, 5, 1659 (1967).

3. B. E. Davydov, B. A. Krentsel, Y. A. Popov, and L. V. Prokofyeva, Vysokomol. Soedin., Ser. A, 5, 321 (1963).

4. S. S. Stivala, G. R. Sacco, and L. Reich, J. Polym. Sci., B, 2, 943 (1964).

5. G. F. D'Alelio and R. K. Schoenig, Rev. Macromol. Sci., 4, 107 (1970).

6. G. F. D'Alelio, J. V. Crivello, R. K. Schoenig, and T. F. Huemmer, J. Macromol. Sci., Chem., A1, 1161 (1967).

7. G. F. D'Alelio, J. V. Crivello, R. K. Schoenig, and T. F. Huemmer, J. Macromol. Sci., Chem., A1, 1259 (1967).

8. G. F. D'Alelio, J. V. Crivello, R. K. Schoenig, and T. F. Huemmer, J. Macromol. Sci., Chem., A1, 1321 (1967).

9. Y. A. Popov, B. E. Davydov, N. A. Kubasova, B. A. Krentsel, and I. I. Konstantinov, Polym. Sci., USSR, 7, 921 (1965).

10. G. F. D'Alelio, J. V. Crivello, R. K. Schoenig, and T. F. Huemmer, J. Macromol. Sci., Chem., A1, 1251 (1967).

11. A. A. Volpe, L. G. Kaufman, and R. G. Dondero, J. Macromol. Sci., Chem., A3, 1087 (1969).

12. L. G. Kaufman, P. T. Funke, and A. A. Volpe, Macromolecules, 3, 358 (1970).

13. A. A. Volpe, L. G. Kaufman, and R. G. Dondero in "Polymers in Space Research," C. L. Segal, Ed., M. Dekker Inc., New York, N. Y., 1970. 